J. Clin. Chem. Clin. Biochem.

Vol 14, 1976, pp. 3-8

\title{
Bildung und Reinigung des Oxidoreductase-Inhibitors aus dem NAD
}

\author{
Von H. Gallati
}

Diagnostische Forschungsabteilung der F. Hoffmann-La Roche \& Co. AG, Basel

(Eingegangen am 21. August/23. Oktober 1975)

Zusammenfassung: Der Oxidoreductase-Inhibitor wird - entgegen der bisher allgemein angenommenen Vermutung nicht aus dem NADH, sondern nur aus dem NAD im alkalischen Milieu gebildet.

NAD-Analoge (z. B. NADP) wie auch NAD-Bauelemente (z. B. ADP) haben auf die Bildung des Inhibitors keinen Einfluß.

Es werden die günstigsten Reaktionsbedingungen für die Bildung des Inhibitors bezüglich pH, Reaktionstemperatur, Inkubationsdauer, Pufferart und NAD-Konzentration untersucht. Die Arbeitsmethode zur Bildung und chromatographischen Isolierung des Oxidoreductase-Inhibitors wird kurz beschrieben.

\section{Formation and purification of the oxidoreductase inhibitor from NAD}

Summary: The oxidoreductase inhibitor is not formed from NADH as previously thought, but only from NAD under alkaline conditions. Analogues of NAD (e.g. NADP) and components of the NAD molecule (e.g. ADP) have no effect on the formation of the inhibitor.

The most favourable $\mathrm{pH}$, temperature, duration of incubation, type of buffer and NAD concentration for the formation of the inhibitor were investigated. The method for the formation and chromatographic isolation of the oxidoreductase inhibitor is briefly described.

\section{Einführung}

Verschiedentlich wurde darauf hingewiesen, daß in den meisten NADH-Präparaten ein Inhibitor enthalten ist (1-11), der in kleinsten Mengen die Aktivitätşbestimmung der Oxidoreductasen signifikant zu stören vermag. Dies wurde vor allem an Hand der diagnostisch wichtigen Lactat-Dehydrogenase (L-lactate: NAD-oxidoreductase, E.C. 1.1.1.27) aufgezeigt $(2,4,6,7,10-12)$.

Der Inhibitor kann chromatographisch vom NADH abgetrennt werden $(4,6,9,10,13)$. Wird aber dieses reine NADH-Präparạt längere Zeit und unter ungünstigen Bedingungen (Feuchtigkeit, alkalisches pH, Licht und höhere Temperatur (5), Einfrieren und Auftauen der alkalischen Coenzymlösung $(2,4,7,8,13)$ ) aufbewahrt, so entsteht der Inhibitor vón neuem.

Die vorliegende Arbeit möchte die Fragen beantworten, aus welcher Substanz der Oxidoreductase-Inhibitor entsteht, welches fuir die Inhibitor-Bildung die günstigsten Reaktionsbedingungen sind und auf welche Weise eine größere Menge Oxidoreductase-Inhibitor hergestellt und gereinigt werden kann.

\section{Material und Methoden}

1. Die Substañzen Adenin, Adenosin, AMP, ADP, ATP, Adenosin-5'-diphospho-Ribose, NAD, NADH, NADP und NADPH wurden von Boehringer Mannheim bezogen. Diese Substanzenreihe wurde ergänzt durch Nicotinsäure (Hoffmann-La Roche) und Nicotinsäureamid (Merck).

2. Für die chromatographische Isolierung des Oxidoreductase-Inhibitors wurde DEAE-Cellulose (Whatmann DE-23) und Sephadex G-15 (Pharmacia) eingesetzt.

Die mit $10 \mathrm{mmol} / 1$ Natriumphosphat-Puffer, pH 8,5 äquilibrierte DEAE-Cellulose wurde in eine Säule $(2,5 \times 40 \mathrm{~cm})$ gepackt und das Substanzgemisch mit dem Inhibitor aufgetragen. Die Fraktionierung erfolgte nach gutem Auswaschen mit einem linearen $\mathrm{NaCl}-$ Gradienten. Sephadex G-15 wurde in $10 \mathrm{mmol} / 1$ Ammoniumcarbonat-Puffer, $\mathrm{pH} 9,8$ aufgenommen und anschließend in eine Säule von $1 \times 180 \mathrm{~cm}$ gepackt. Alle chromatographischen Arbeiten wurden bei Raumtemperatur durchgefuihrt.

3. Die Bedingungen zur Bildung des Inhibitors werden für jeden einzelnen Versuch speziell angegeben.

4. Die Menge des gebildeten Inhibitors kann nicht exakt angegeben werden, weil dessen Bestimmung nur aufgrund der Aktivitätshemmung einer Oxidoreductase erfolgen kann. Dabei ist zu berücksichtigen, daß die einzelnen Oxidoreductasen durch die gleiche Menge Inhibitor sehr unterschiedlich gehemmt werden (7). 
Zur Abschätzung der gebildeten Menge Inhibitor haben wir die Aktivitätshemmung der Lactat-Dehydrogenase (EC 1.1.1.27) aus Rinderherz (Boehringer) bestimmt.

Dazu wurden zu

2,6 ml Lactat-Dehydrogenase-Testlösung, deren Zusammensetzung den Empfehlungen der Deutschen Enzymkommission entsprach (14)

$0,1 \mathrm{ml}$ Lactat-Dehydrogenase-Lösung (280-320 U/1) und

$0,05 \mathrm{ml}$ Probe in entsprechender Verdünnung zugegeben.

5. Die Ammoniumionen-Konzentration wurde nach der modifizjerten Berthelot-Reaktion (15), anorganisches Phosphat nach der Malachitgrün-Methode (16), AMP und ADP enzymatisch nach der Methode von Adam (17) bestimmt.

\section{Ergebnisse}

Ausgangssubstanz für die Bildung des Oxidoreductase-Inhibitors

Der Oxidoreductase-Inhibitor ist nicht identisch mit einem Bauelement des NADH noch mit dessen oxydierter (NAD) oder phosphorylierter Form (NADP, NADPH). Adenin, Adenosin, AMP, ADP, ATP', Adenosin-5'-diphospho-Ribose, Nicotinsäure, Nicotinsäureamid und auch die NADH-Analogen NAD, NADP und NADPH haben im fraglichen Konzentrationsbereich (bis $0,2 \mathrm{mmol} / \mathrm{l}$ ) keinen hemmenden Einfluß auf die Aktivität der Lactat-Dehydrogenase.

Um festzustellen, ob der Inhibitor direkt aus dem $\mathrm{NADH}$, aus einem NADH-Analogen oder einem NADHBauelement gebildet wird, wurden die oben angeführten Substanzen in einer Konzentration von $10 \mathrm{mmol} / \mathrm{l}$ einerseits in $0,1 \mathrm{~mol} / 1 \mathrm{HCl}$ und anderseits in $0,1 \mathrm{~mol} / 1 \mathrm{NaOH}$ während 24 Stunden bei $+45^{\circ} \mathrm{C}$ in verschlossenen Glasampullen inkubiert.

Eine Inhibitorbildung konnte nur in den alkalischen Lösungen des NADH, des NAD und des NADP festgestellt werden (Tab. 1). Die stark unterschiedliche Menge an gebildetem Imhibitor in diesen drei Lösungen läßt vermuten, daß das NAD als Ausgangssubstanz für die Bildung des Inhibitors angesehen werden muß, und daß die Inhibitorbildung in den alkalischen NADH- und NADP-

Tab. 1. Bildung des Oxidoreductase-Inhibitors in alkalischer NAD-, NADH-, NADP- und NADPH-Lösung. Einzelheiten im Text.

Zur Aktivitätsbestimmung der Lactat-Dehydrogenase wurde zur Testlösung $75 \mu \mathrm{mol} / 1$ des entsprechenden inkubierten Coenzyms zugegeben und der Grad der Aktivitätshemmung bestimmt.

\begin{tabular}{llll}
\hline Coenzym & $\begin{array}{l}\text { Farbe der inku- } \\
\text { bierten Coen- } \\
\text { zymlösung }\end{array}$ & $\begin{array}{l}\text { Fluoreszenz der Grad der Aktivi- } \\
\text { inkubierten Co- tätshemmung } \\
\text { enżymlösung } \\
\text { Anregung } \\
\text { der Lactat- } \\
\text { Dehydrogenase }\end{array}$ \\
\hline NAD & gelb-braun & ++++ & $75 \%$ \\
NADH & leicht gelb & ++ & $11 \%$ \\
NADP & gelb-braun & +++ & $5 \%$ \\
NADPH & leicht gelb & ++ & $0 \%$ \\
\hline
\end{tabular}

Lösungen auf das NAD zurückzufuihren ist, das als Verunreinigung oder durch Autoxydation des NADH in diesen beiden Lösungen enthalten ist.

Um dieș genauer abzuklären, warden $10 \mathrm{mmol} / \mathrm{l}$ Coenzym-Lösungen in $0,1 \mathrm{~mol} / 1 \mathrm{NaOH}$ hergestellt und dabei das Mischungsverhältnis von NAD und NADH variiert. Nach $1 \mathrm{Tag}$ Inkubation bei $45^{\circ} \mathrm{C}$ in verschlossenen Glasampullen wurde die gebildete Menge Inhibitor in den Lösungen bestimmt. Die Resultate, die in der Tabelle 2 zusammengestellt sind, zeigen, daß die Menge des gebildeten Inhibitors mit steigendem Gehalt an NAD in der Coenzymmischung zunimmt, und sie bestätigen die Vermutung, daß der Oxidoreductase-Inhibitor primär aus dem NAD gebildet wird.

Das NAD muß vorschriftsgemäß im sauren Milieu aufbewahrt werden, da diese Substanz bekannterweise im alkalischen pH-Bereich sehr instabil ist und rasch zum Nicotinsäureamid und zur Adenosin-5' -diphospho-Ribose abgebaut wird, die ihrerseits zu ADP und AMP übergeht $(17,18)$.

In einem weiteren. Versuch wurde NAD und parallel dazu die NAD-Abbauprodukte Adenosin-5'-diphosphoRibose, ADP, Ribose und Nicotinsäureamid in verschiedenen Vàriationsmöglichkeiten in einer.Konzentration von jeweils $10 \mathrm{mmol} / 1$ in $0,1 \mathrm{~mol} / 1 \mathrm{NaOH}$ während 24 Stunden bei $45^{\circ} \mathrm{C}$ inkubiert. Der Oxidoreductase-Inhibitor wurde nur in der alkalischen NAD-Lösung gebildet, was zeigt, daß er weder ein Kondensationsprodukt der abgespaltenen NAD-Bauelemente ist noch aus diesen entstehen kann.

Einfluß der NAD-Konzentration auf die Bildung des Oxidoreductase-Inhibitors

Verschiedene NAD-Konzentrationen wurden in $0,2 \mathrm{mmol} / 1$ Phosphatpuffer, $\mathrm{pH} 11$ während $1 \mathrm{Tag}$ bei $45^{\circ} \mathrm{C}$ inkubiert, um abzuklären, ob die Konzentration auf die Bildung des Inhibitors einen Einfluß ausübt. Anschließend an die Inkubation wurden die einzelnen Lösungen mit entsprechenden Mengen Phosphatpuffer verdünnt, so daß für die Bestimmung des Inhibitors gleichmolare NAD-Lösungen entstanden. Die Resultate sind in Tabelle 3 aufgeführt. Sie zeigen eine deutliche Zunahme der Inhibitorbildung bei höherer NAD-Konzentration.

\section{Einfluß des Puffersystems auf die Bildung des} Oxidoreductase-Inhibitors

Um den Einfluß des Puffersystems auf die Bildung des Inhibitors abzuklären, wurden in Ampullenflaschen je $10 \mathrm{mg}$ festes NAD und $20 \mathrm{mg}$ der betreffenden Puffersubstanz eingewogen. Um einen hohen pH-Wert und zugleich genügend Feuchtigkeit zu garantieren, wurde $\mathrm{zu}$ dem Substanzgemisch $0,1 \mathrm{ml} 1 \mathrm{~mol} / 1 \mathrm{NaOH}$ zupipettiert. Die Ampullen wurden bei $45^{\circ} \mathrm{C}$ während 1. Tag inkubiert. Zur Bestimmung des Inhibitorgehaltes in den einzelnen Ansätzen wurden die entsprechenden Löșungen durch 
Tab. 2. Bildung des Oxidoreductase-Inhibitors bei unterschiedlichem Mischungsverhältnis von NAD und NADH in alkalischer Lösung. Einzelheiten im Text.

Zur Aktivitätsbestimmung der Lactat-Dehydrogenase wurden zur Testlösung $75 \mu \mathrm{mol} / 1$ der entsprechend inkubierten Coenzymlösung (NAD/NADH) zugegeben und der Grad der Aktivitätshemmung bestimmt.

\begin{tabular}{|c|c|c|c|c|}
\hline $\begin{array}{l}\text { Mischur } \\
\text { NADH }\end{array}$ & NAD & $\begin{array}{l}\text { Farbe der inkubierten } \\
\text { Coenzymlösung }\end{array}$ & $\begin{array}{l}\text { Fluoreszenz der } \\
\text { inkubierten Coenzym- } \\
\text { lösung Anregung: } 366 \mathrm{~nm}\end{array}$ & $\begin{array}{l}\text { Grad der Aktivitäts- } \\
\text { hemmung der Lactat- } \\
\text { dehydrogenase [\%] }\end{array}$ \\
\hline $\begin{array}{r}100 \\
90 \\
80 \\
60 \\
50 \\
40 \\
20 \\
10 \\
-\end{array}$ & $\begin{array}{r}- \\
10 \\
20 \\
40 \\
50 \\
60 \\
80 \\
90 \\
100\end{array}$ & $\begin{array}{l}\text { leicht gelb } \\
\text { leicht gelb } \\
\text { leicht gelb } \\
\text { gelb } \\
\text { gelb } \\
\text { orange } \\
\text { orange } \\
\text { orange } \\
\text { gelb-braun }\end{array}$ & $\begin{array}{l}++ \\
++ \\
++ \\
+++ \\
+++ \\
+++ \\
++++ \\
++++ \\
++++\end{array}$ & $\begin{array}{r}9 \\
15 \\
24 \\
36 \\
42 \\
50 \\
63 \\
70 \\
77\end{array}$ \\
\hline
\end{tabular}

Tab. 3. Einfluß der NAD-Konzentration während der Inkubation auf die Bildung des Oxidoreductase-Inhibitors. Einzelheiten im Text.

Zur Aktivitätsbestimmung der Lactat-Dehydrogenase wurden zur Testlösung $30 \mu \mathrm{mol} / 1$ des inkubierten NAD zugegeben und der Grad der Aktivitätshemmung bestimmt.

NAD-Konzentration während der Inkubation [mmol/1]

\begin{tabular}{ll} 
& genase [\%] \\
\hline 50 & 90 \\
25 & 65 \\
12,5 & 52 \\
6,25 & 45 \\
3,125 & 35 \\
1,56 & 18 \\
0,78 & 15 \\
\hline
\end{tabular}

Tab. 4. Einfluß des Puffersystems auf die Bịldung des Oxịioreductase-Inhibitors.

Einzelheiten im Text.

Zur Aktivitätsbestimmung der Lactat-Dehydrogenase wurden żur Testlösung je $15 \mu \mathrm{mol} / 1$ des inkubierten NAD zugegeben und der Grad der Aktivitätshemmung bestimmt.

\begin{tabular}{|c|c|c|}
\hline $\begin{array}{l}\text { Puffersystem, in dem } \\
\text { das NAD inkubiert } \\
\text { wurde }\end{array}$ & $\begin{array}{l}\text { pHi Wert der verdünn- } \\
\text { ten Lösung }\end{array}$ & $\begin{array}{l}\text { Grad der Aktivi- } \\
\text { tätshemmung } \\
\text { der Lactat-Dehy- } \\
\text { drogenase [\%] }\end{array}$ \\
\hline $\begin{array}{l}\mathrm{NaOH} \\
\text { Triäthanolamin } / \mathrm{NaOH} \\
\mathrm{Na}_{2} \mathrm{CO}_{3} / \mathrm{NaOH} \\
\mathrm{Triis} / \mathrm{NaOH}^{2} \\
\mathrm{Na}_{3} \mathrm{PO}_{4} / \mathrm{NaOH} \\
\mathrm{Na}_{2} \mathrm{SO}_{4} / \mathrm{NaOH} \\
\mathrm{Natriumoxalat}_{\mathrm{NaOH}} \\
\mathrm{Natriumtetraborat} / \\
\mathrm{NaOH}\end{array}$ & $\begin{array}{r}11,24 \\
11,30 \\
10 ; 20 \\
11,05 \\
11,46 \\
10,87 \\
10 ; 97 \\
9,73\end{array}$ & $\begin{array}{r}15 \\
22 \\
71 \\
47 \\
76 \\
20 \\
51 \\
0\end{array}$ \\
\hline
\end{tabular}

Zugabe von je $5 \mathrm{ml}$ dest. Wasser verdünnt. Die Resultate dieses Versuches sind in Tabelle 4 zusammengestellt.

Die unterschiedlichen pH-Werte in den einzelnen inkubierten NAD-Proben können die erhebliche Mengendifferenz des gebildeten Oxidoreductase-Inhibitors nicht erklären. Es ist vielmehr anzunehmen, daß die einzelnen Puffersubstanzen die Inhibitorbildung unterschiedlich beeinflussen, wobei vor allem $\mathrm{Na}_{2} \mathrm{CO}_{3}$ und $\mathrm{Na}_{3} \mathrm{PO}_{4}$ die Bildung des Inhibitors begünstigen.

Bildung des Oxidoreductase-Inhibitors in Abhängigkeit vom $\mathrm{pH}$-Wert

Um die' pH-Abhängigkeit zu untersuchen, sind $10 \mathrm{mmol} / 1$ NAD-Lösungen in 0,2 mol/l Kaliumphosphatpuffer, dessen pH-Wert zwischen 6 und 13 variiert wurde, in verschlossenen Ampullen bei $+45^{\circ} \mathrm{C}$ während 2 bzw. 24 Stunden inkubiert worden. Anschließend wurden die einzelnen Lösungen durch Zugabe von dest. Wasser verdünnt und die gebildete Menge Inhibitor bestimmt. Die Resultate, die in Tabelle 5 zusammengefaßt sind, zeigen, daß die Inhibitorbildung mit höherem pH-Wert zwar beschleunigt wird, daß aber anderseits in zu alkalischem Milieu die Stabilität des Inhibitors offenbar abnimmt.

Abhängigkeit der Bildung des Inhibitors von der Reaktionsdauer und der Inkubationstemperatur

Um diese Abhängigkeit zu studieren, wurde eine $10 \mathrm{mmol} / 1 \mathrm{NAD}$-Lösung in $0,1 \mathrm{~mol} / 1 \mathrm{~K}_{3} \mathrm{PO}_{4}$-Puffer, pH 10,5 in 5 Ampullen aufgeteilt und je eine bei den Temperaturen $2-8^{\circ} \mathrm{C}$, Raumtemperatur, $35^{\circ} \mathrm{C}, 45^{\circ} \mathrm{C}$ und $60^{\circ} \mathrm{C}$ inkubiert. Nach bestimmten Zeitintervallen wurde von den betreffenden Lösungen eine Probe entnommen und darin der Gehalt an Oxidoreductase-Inhibitor bestimmt. Die Resultate der Tabelle 6 zeigen, daß mit erhöhter Inkubationstemperatur zwar die Bildung des Inhibitors beschleunigt wird, daß sich aber anderseits auch die Hitze-Instabilität des Inhibitors bemerkbar macht. 
Tab. 5. Einfluß des pH-Wertes der inkubierten NAD-Lösung auf die Bildung des Oxidoreductase-Inhibitors.

Einzelheiten im Text.

Zur Aktivitätsbestimmung der Lactat-Dehydrogenase wurde zur Testlösung je $15 \mu \mathrm{mol} / \mathrm{l}$ des inkubierten NAD zugegeben und der Grad der Aktivitätshemmung bestimmt.

\begin{tabular}{llc}
\hline $\begin{array}{l}\text { pH-Wert der inkubierten } \\
\text { NAD-Lösung }\end{array}$ & \multicolumn{2}{l}{$\begin{array}{l}\text { Grad der Aktivitätshemmung der } \\
\text { Lactat-Dehydrogenase [\%] nach } \\
\text { einer Inkubationsdauer des NAD } \\
\text { von } \\
2 \mathrm{~h}\end{array}$} \\
\hline 6,4 & $24 \mathrm{~h}$ \\
7,5 & 0 & 0 \\
8,6 & 10 & 24 \\
9,6 & 21 & 48 \\
10,5 & 28 & 64 \\
11,5 & 49 & 76 \\
12,4 & 59 & 64 \\
\hline
\end{tabular}

Tab. 6. Einfluß der Inkubationstemperatur und der Inkubationsdauer der alkalischen NAD-Lösung auf die Bildung des Oxidoreductase-Inhibitors.

Einzelheiten im Text.

Zur Aktivitätshemmung der Lactat-Dehydrogenase wurde zur Testlösung je $5 \mu \mathrm{mol} / 1$ des inkubierten NAD zugegeben und der Grad der Aktivitätshemmung bestimmt.

\begin{tabular}{|c|c|c|c|c|c|}
\hline \multirow{2}{*}{$\begin{array}{l}\text { NAD-Inku- } \\
\text { bations- } \\
\text { Dauer [h] }\end{array}$} & \multicolumn{5}{|c|}{$\begin{array}{l}\text { Grad der Aktivitätshemmung der Lactat-Dehydro } \\
\text { genase bei der NAD-Inkubationstemperatur: }\end{array}$} \\
\hline & $2-8^{\circ} \mathrm{C}$ & $\begin{array}{l}\text { Raum- } \\
\text { tempera- } \\
\text { tur }\end{array}$ & $35^{\circ} \mathrm{C}$ & $45^{\circ} \mathrm{C}$ & $60^{\circ} \mathrm{C}$ \\
\hline 1 & - & - & - & 11 & 19 \\
\hline 2 & - & - & - & 25 & 30 \\
\hline 4 & - & - & 28 & 35 & 48 \\
\hline 6 & - & - & 35 & 40 & 20 \\
\hline 24 & 12 & 40 & 34 & 25 & 12 \\
\hline 48 & 32 & 46 & 39 & 20 & 13 \\
\hline
\end{tabular}

In diesem Zusammenhang wurde auch der zeitliche Verlauf der Bildung des Oxidoreductase-Inhibitors genauer verfolgt, indem eine $8 \mathrm{mmol} / 1 \mathrm{NAD}$-Lösung in $0,1 \mathrm{~mol} / 1$ $\mathrm{NaOH}$ im Wasserbad bei $60^{\circ} \mathrm{C}$ inkubiert wurde.

Nach bestimmten Zeitintervallen wurde von der NADLösung eine Probe entnommen und darin neben dem Gehalt an Inhibitor zugleich die Menge an abgespaltenen Ammonium- und Phosphationen bestimmt. Die dabei erhaltenen Resultate sind in Abbildung 1 graphisch dargestellt.

Die Ammoniumionen stammen mit großer Wahrscheinlichkeit vom Nicotinsäureamidanteil des NAD, denn Parallelversuche haben ergeben, daß von Adenin, Adenosin, AMP, ADP und Adenosin-5'-diphospho-Ribose unter gleichen Bedingungen keine Ammoniumionen freigesetzt werden. Wird aber Nicotinsäureamid in $0,1 \mathrm{~mol} / 1$ $\mathrm{NaOH}$ bei $60^{\circ} \mathrm{C}$ inkubiert, steigt der Gehalt an Ammoniumionen sehr schnell an.

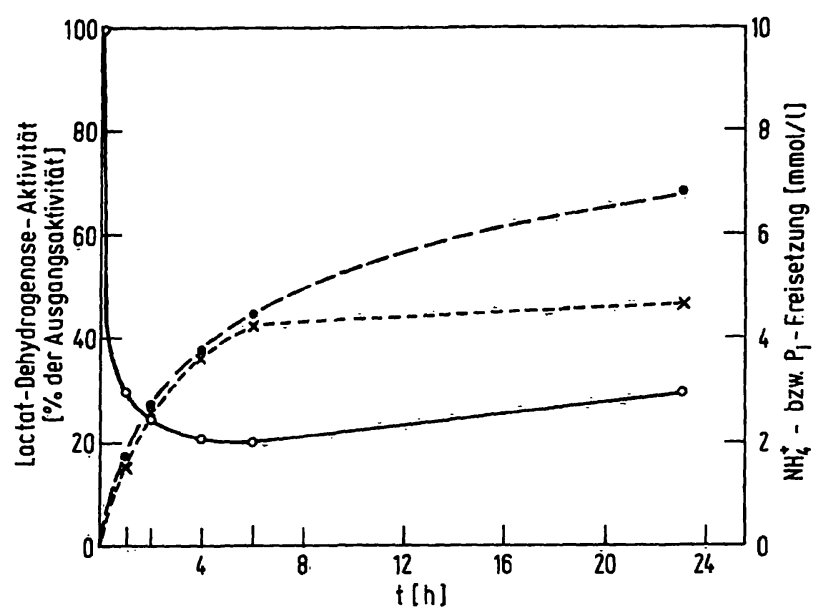

Abb. 1. Zeitlicher Verlauf der Bildung des Oxidoreductase-Inhibitors $(0-0)$ und der Freisetzung von Ammonium$(\cdot--)$ und Phosphationen $(x---x) .8 \mathrm{mmol} / \mathrm{l}$ NADLösung wurde in $0,1 \mathrm{~mol} / 1 \mathrm{NaOH}$ bei $60^{\circ} \mathrm{C}$ inkubiert. Nach bestimmten Zeitintervallen wurde von dieser Lösung eine kleine Probe entnommen, um damit die drei genannten Bestimmungen durchzufuhren. Zur Messung der Aktivitätshemmung der Lactat-Dehydrogenase wurde der Testlösung $15 \mu \mathrm{mol} / 1$ inkubiertes NAD zugefügt.

Anreicherung des NAD während der Inkubation zur Bildung des Inhibitors mit verschiedenen NAD-Bauelementen

Bei der Bildung des Inhibitors könnte ein Teil des NAD in seiner chemischen Struktur umgeformt werden und anschließend zusammen mit Abbauprodukten des NAD ein Kondensationsprodukt bilden. Um diese Möglichkeit abzuklären, wurden zu einer $10 \mathrm{mmol} / 1$ NAD-Lösung in $0,1 \mathrm{~mol} / 1 \mathrm{Na}_{3} \mathrm{PO}_{4}$-Puffer $\mathrm{pH} 11,0$ von dẹn einzelnen NAD-Bauelementen je $5 \mathrm{mmol} / \mathrm{l}$ zugefügt, und während $1 \mathrm{Tag}$ bei $45^{\circ} \mathrm{C}$ inkubiert. Der Gehalt an gebildetem Inhibitor konnte durch keines der zugesetzten NAD-Bauelemente signifikant erhöht werden.

Arbeitsmethode zur Bildung und chromatographischen Isolierung des Oxidoreductase-Inhibitors unter Berücksichtigung der günstigsten Reaktionsbedingungen

\section{Bildung des Inhibitors}

$1,0 \mathrm{~g} \beta$-NAD (oxidierte Form, Boehringer Nr. 15300, $\mathrm{MG}=663,4$, Gehalt an NAD $=90 \%$ ) werden mit

$2,0 \mathrm{~g} \mathrm{~K}_{3} \mathrm{PO}_{4} \cdot 7 \mathrm{H}_{2} \mathrm{O}($ Merck Nr. 5103, $\mathrm{MG}=338,38)$ in einer Glasampulle gut verrieben und während 3 Tagen bei Raumtemperatur in wassergesättigter Atmosphäre inkubiert.

Die Farbe der Substanzmischung ändert sich während der Inkubationsdauer von weiß nach schwarz, und es entsteht eine dickflüssige Masse.

Nach der Inkubation wird das Substanżgemisch durch Zugabe von $100 \mathrm{ml}$ dest. Wasser gelöst. Bezogen auf das eingesetzte $\operatorname{NAD}(90 \%=0,9 \mathrm{~g})$ hat diese Lösung eine 
Konzentration von 13,56 mmol/1. Die Lösung ist dunkelbraun gefärbt und zeigt bei einer Anregung von $366 \mathrm{~nm}$ eine starke gelbgrüne Fluoreszenz. Der pH-Wert liegt bei 10,9 .

Die Aktivität der Lactat-Dehydrogenase wird zu 50\% gehemmt, wenn zur Testlösung $5 \mu \mathrm{mol} / 1$ des inkubierten NAD zugefügt wird.

Auf Grund der Bestimmung von ADP und AMP kann gesagt werden, daß unter diesen Inkubationsbedingungen etwa $65 \%$ des eingesetzten NAD zum AMP und etwa $20 \%$ zum ADP abgebaut werden.

\section{Säulenchromatographische Reinigung des Inhibitors}

Ein Teil dieser braunen, inhibitorreichen Lösung wird auf DEAE-Cellulose aufgetragen, die mit $10 \mathrm{mmol} / \mathrm{l}$ Natriumphosphatpuffer vom $\mathrm{pH} 8,5$ äquilibriert und in eine Säule $(2,5 \times 40 \mathrm{~cm})$ gepackt wurde.

Nach dem Auftragen der Probe wird die Säule mit dem gleichen Puffer gut durchgewaschen, und anschließend die adsorbierte Substanz mit Hilfe eines linearen $\mathrm{NaCl}$ Gradienten fraktioniert. Chromatogramm in Abb. 2.

Die Fraktionen mit dem Inhibitor werden gepoolt, eingeengt, und auf Sephadex G-15, das in $10 \mathrm{mmol} / 1 \mathrm{Ammo}-$ niumcarbonatpuffer vom $\mathrm{pH} 9,8$ aufgenommen und in eine Säule $(1 \times 180 \mathrm{~cm})$ gepackt wurde, aufgetragen. Chromatogramm in Abbildung 3.

Die Fraktionen mit dem Inhibitor werden gepoolt. Die Lösung kann unter leichtem Vakuum bei $40^{\circ} \mathrm{C}$ auf das gewünschte Volumen eingeengt und bei $2-8^{\circ} \mathrm{C}$ im alkalischen Milieu über längere Zeit ohne nennenswerten Aktivitätsverlust des Inhibitors aufbewahrt werden.

Die Aktivität der Lactat-Dehydrogenase kann zu 50\% gehemmt werden, wenn der Testlösung $0,55 \mu \mathrm{mol} / 1$ des gereinigten Oxidoreductase-Inhibitors zugefügt wird. Der

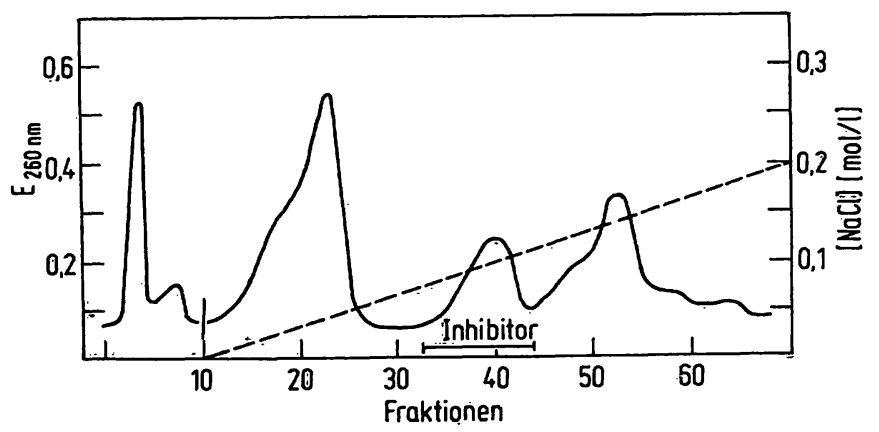

Abb. 2. Säulenchromatographische Trennung des OxidoreductaseInhibitors: DEAE-Cellulose, äquilibriert mit $10 \mathrm{mmol} / 1$. Phosphatpuffer, pH 8,5, wurde in eine Säule von $2,5 \times 40 \mathrm{~cm}$ gepackt. Ein Teil der inhibitorreichen Lösung wurde aufgetragen, und nach gutem Auswaschen die adsorbierten Substanzen mit einem linearen $\mathrm{NaCl}$ Gradienten (-- ) fraktioniert. Die Extinktion des Eluates wurde bei der Wellenlänge $260 \mathrm{~nm}(-)$ gemessen. Volumen der einzelnen Fraktion $=10 \mathrm{ml}$.

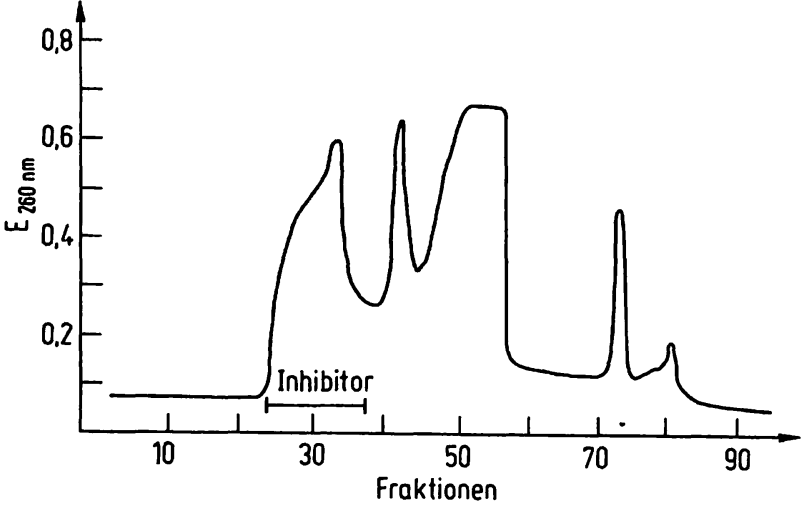

Abb. 3. Säulenchromatographische Trennung des Oxidoreductase-Inhibitors: Sephadex G-15, aufgenommen in $10 \mathrm{mmol} / \mathrm{l}$ Ammoniumcarbonatpuffer vom $\mathrm{pH} 9,8$, wurde in eine Säule von $1 \times 180 \mathrm{~cm}$ gepackt, anschließend ein Teil der Inhibitorlösung aufgetragen, die vorher auf DEAE-Cellulose gereinigt und konzentriert wurde. Die Extinktion des Eluates wurde bei der Wellenlänge $260 \mathrm{~nm}$ gemessen. Das Volumen der einzelnen Fraktionen betrug $2,5 \mathrm{ml}$.

Konzentrationsangabe des Inhibitors liegt dabei die hypothetische Annahme zugrunde, daß der molare Extinktionskoeffizient für NAD und für den Inhibitor bei der Wellenlänge $260 \mathrm{~nm}$ identisch ist.

\section{Diskussion}

Die in dieser Arbeit mitgeteilten Resultate zeigen, daß der Inhibitor aus dem NAD im alkalischen Milieu gebildet wird, und daß er weder ein Kondensations- noch ein Abbauprodukt von bestimmten NAD-Bauelementen darstellt.

Da das NAD nur im sauren Milieu stabil ist und folglich stets als freie Säure aufbewahrt wird, ist es verständlich, daß in den NAD-Präparaten der Oxidoreductase-Inhibitor fehlt (9).

Anderseits ist das NADH in seiner reduzierten Form nur im alkalischen Milieu beständig. Wie aus den verschiedenen Versuchen ersichtlich ist, kann sich der Inhibitor zwar nicht aus dem NADH bilden. Es ist aber anzunehmen, daß in den meisten kommerziellen NADH-Präparaten ein größerer oder kleinerer Anteil an NAD enthalten ist, teils wegen ungenügender Reinigung, teils auch durch Autoxydation des NADH während der Konfektionierung und Lagerzeit. Tritt nun Sauerstoff und Feuchtigkeit zum NADH-Präparat, so sind die Bedingungen gegeben, daß einerseits weiteres NADH oxydiert wird, und daß anderseits aus dem vorhandenen und neu gebildeten NAD der Inhibitor entstehen kann. Dabei ändert sich die weiße Farbe des NADH-Salzes in Richtung gelborange-braun, je nach Grad der Zersetzung.

Die Resultate über die Abhängigkeit der Inhibitorbildung von der NAD-Konzentration erklärt auch, warum in 
feuchten NADH-Präparaten mehr Inhibitor gebildet wird als in verdünnten NADH-Löslingen.

NADH in alkalischer Lösung ist nur beschränkte Zeit haltbar, bedingt einerseits wegen der Instabilität der reduzierten Form, und anderseits wegen der fortschreitenden Bildung des Inhibitors. Lowry (19) hat vorgeschlagen, das NADH in einer Konzentration, die $5 \mathrm{mmol} / 1$ nicht übersteigt, in Natriumcarbonatlösung $\mathrm{pH} 10$ bei $4^{\circ} \mathrm{C}$ aufzubewahren. Demgegenüber haben Strandjord (4) und Gerhardt (11) beste Stabilitätsresultate des gelösten $\mathrm{NADH}$ in $50 \mathrm{mmol} / \mathrm{l}$ Tris/HCl-Puffer, $\mathrm{pH} \mathrm{7,4} \mathrm{erhalten.}$ Auf Grund unserer Untersuchungen kann man in Hinsicht auf eine weitgehende Unterbindung der Inhibitorbildung folgende Forderungen für die NADH-Lösung aufstellen:
- Das NADH-Präparat muß möglichst frei von NAD sein und keine oxydierenden Substanzen enthalten.

- Die NADH-Konzentration soll möglichst niedrig gehalten werden.

- Als Puffer, sofern überhaupt erforderlich, darf kein Phosphat, Carbonat oder Oxalat eingesetzt werden.

- Der pH-Wert der NADH-Lösung muß so tief als möglich gewählt werden. Der limitierende Faktor bildet dabei die Stabilisierung der reduzierten Form des NADH.

- Die Lösung soll bei $2-8^{\circ} \mathrm{C}$ aufbewahrt werden.

- Es darf nur soviel NADH aufgelöst werdèn, daß die Lösung in möglichst kurzer Zeit aufgebraucht wird.

\section{Literatur}

1. Dalziel, K. (1962), Biochem. J. 84, 240-244.

2. Fawcett, C., Ciotti, M. \& Kaplan, N. (1961), Biochim. Biophys. Acta 54, 210-212.

3. Dalziel, K. (1963), J. Biol. Chem. 238, 1538-1543.

4. Strandjord, P. \& Clayson, K. (1966), J. Lab. Clin. Med. 67, 144-153.

5. Holman, M., Willis, J. \& Siegel, J. (1966), Clin. Chem. 12, 543.

6. McComb, R. \& Gay, R. (1968), Clin. Chem. 14, 754-763.

7. Koltzsch, S. \& Klotzsch, H. (1969), Clin. Chem. 15, 10561061.

8. Fine, I., Fawcett, C., Everse, J. \& Kaplan, N. (1962), J. Amer. Chem. Soc. (Abstr.) $55 \mathrm{C}$.

9. Babson, A. \& Arndt, E. (1970), Clin. Chem. 16, 254-255.

10. Berry, A., Lott, J. \& Grannis, G. (1973), Clin. Chem. 19, $1255-1258$
11. Gerhardt, W., Kofoed, B., Westlund, L. \& Pavlu, B. (1974), Scand. J. Clin. Lab. Invest 33, 3-51.

12. Härtel, A., Helger, R. \& Lang, H. (1968), diese Z. 6, 259-262.

13. Silverstein, E. (1965), Anal. Biochem. 12, 199-212.

14. Deutsche Gesellschaft für Klinische Chemie (1972), diese Z. 10, 182-192.

15. Richterich, R. (1968), „Klinische Chemie-Theorie und Praxis“, Karger, 2. Aufl. Basel.

16. Bastiaanse, A. \& Meijers, C. (1968), diese Z. 6, 48-51.

17. Adam, H. (1970), (Bergmeyer, H. ed), Methoden der enzymatischen Analyse, 2. Aufl. S. 2051, Verlag Chemie, Weinheim.

18. Hilvers, A. \& van Dam, K. (1964), Biochim. Biophys. Acta 81, 391-394.

19. Lowry, O., Passonneau, J. \& Rock, M. (1961), J. Biol. Chem. $236,2756-2759$.
Dr. H. Gallati,

Diagnostische Forschungsabteilung, F. Hoffmann-La Roche \& Co. AG, Grenzacherstr. 124 CH-4002 Basel, Schweiz 